\title{
CIENCIA\&SALUD
}

\section{Definición y manejo inicial del Trauma craneoencefálico leve.}

\section{Definition and management of mild traumatic brain injury.}

Rebeca Alina Watson Hernandez ', Rebeca Soto Rodríguez²

1 y 2 Médico general. Trabajador independiente, Heredia Costa Rica

Contactos: rebewatsonhdez_@hotmail.com rebecasotoro@gmail.com

\section{RESUMEN}

El trauma craneoencefálico es una de las consultas más frecuentes del Servicio de Emergencias. Este se divide en leve, moderado y severo según la escala de Glasgow, sin embargo, predomina el trauma leve. Los principales síntomas suelen ser pérdida de consciencia, bradipsiquia y amnesia. Estos pueden persistir por un par de días o incluso meses en lo que se conoce como síndrome post concusivo. No todo paciente con TCE (Trauma Craneoencefálico) leve amerita estudios de imagen, ya que esto sería una exposición innecesaria a la radiación y se daría un uso inadecuado a esta herramienta tan importante. Se han creado escalas como guías para decidir a quién es verdaderamente necesario realizar una TC con el fin de no hacer un uso indiscriminado ni saturar el servicio de Emergencias. Entre estas escalas se encuentran la Regla de Tomografía de SNC de Canadá y la escala de Nueva Orleans. A su vez, se deben considerar patologías concomitantes como coagulopatías, mecanismo de lesión y riesgo de lesiones intracraneales para decir si el paciente debe quedarse o no en observación: de lo contrario podrá ser egresado con recomendaciones y signos de alarma.

Palabras Clave: Trauma craneoencefálico, escala de Glasgow, leve.

\section{Cómo citar:}

Watson Hernandez, R. A., \& Soto Rodríguez, R. Trauma craneoencefálico leve. Revista Ciencia Y Salud Integrando Conocimientos, 5(5), Pág. 57-64. https:// doi.org/10.34192/ cienciaysalud.v5i5.336

Recibido: 02/jul/2021

Aceptado: 12/oct/2021

Publicado: 18/oct/2021

\section{ABSTRACT}

Trauma brain injury is one of the most frequent consultations in the Emergency Department. It is divided into mild, moderate and severe according to the Glasgow scale. Mild traumatic brain injury is the one that predominates. The main symptoms are usually loss of consciousness, bradipsichia and amnesia. These symptoms can persist for a couple of days in what is known as Post concussion Syndrome. Not every patient with mild TBI (Traumatic Brain Injury) require imaging studies as this would be an unnecessary exposure to radiation and a very important tool would be misused. Scales have been created as guides to decide to whom is necessary to perform a CT (Computed Tomography) for not making an indiscriminate use or saturate the Emergency service. These scales include the Canada CNS (Central Nervous System) Tomography Rule and the New Orleans scale. At the same time, concomitant pathologies such as coagulopathies, mechanism of injury and risk of intracranial injuries must be considered to determine whether or not the patient should remain under observation. Otherwise, they may be discharged with recommendations and warning signs.

Keywords: Trauma brain injury, Glasgow scale, mild. 


\section{INTRODUCCIÓN}

El trauma craneoencefálico es una de las consultas más frecuentes en el Servicio de Emergencias. Predomina en niños ( $0-4$ años), adolescentes y adultos jóvenes (15 - 24 años) y en ancianos (> 65 años) (1). En niños abarca el $3-5 \%$ de las consultas del SEM (Servicio de Emergencias) y de ellos un 90\% son traumas leves (2).

La clasificación más utilizada para TCE (trauma craneoencefálico) se basa en la escala de coma de Glasgow. Los pacientes con una puntuación de 13 a 15 (30 minutos posterior al trauma) se clasifican como TCE leve. Los que tengan una puntuación de 9 a 12 moderado y menor o igual a 8 como grave (3). La mayor parte de pacientes cuando llegan al SEM (servicio de emergencias) tienen una puntuación de 14 y 15 puntos en la Escala de Glasgow (4). Sin embargo, existe una tendencia de excluir a pacientes con 13 puntos de la categoría leve, ya que existe una gran probabilidad de que la TC (tomografía computarizada) del SNC (sistema nervioso central) presente alteraciones, por lo que una puntuación de 13 se encuentra más cerca del TCE moderado (5).

Es importante tener presente que entre del $1 \%$ al $4 \%$ de las personas que padecen TCE leve desarrollan complicaciones que pueden aumentar la morbimortalidad (6). Dada su alta incidencia y bajo porcentaje de presentar complicaciones se deben considerar los criterios para realizar estudios de imagen más complejos como TC SNC para no exponer al paciente de forma innecesaria a radiación ni saturar el servicio de emergencias.

\section{Definiciones}

Hay que reconocer que existen diferencias entre los términos de un TCE leve y una contusión cerebral. Aunque generalmente se usen como sinónimos, tienen distintas connotaciones para los pacientes y profesionales de la salud (7). Además, entre los TCE leves, la presentación más frecuente suele ser la contusión (8).

- Trauma craneoencefálico: perturbación fisiológica de la función cerebral debido a una lesión traumática que se manifiesta por al menos una de las siguientes características (1):

- Cualquier período de pérdida de consciencia.

- Cualquier episodio de pérdida de la memoria con respecto a los episodios ocurridos inmediatamente antes o después del evento.

- Alteración del estado mental al momento del trauma.

- Déficit neurológico focal que puede o no ser transitorio, pero sin pérdida de consciencia mayor a 30 min; clasificación en la escala de Glasgow menor a 13 puntos y amnesia postraumática mayor de 24 horas.

- Contusión cerebral: proceso fisiopatológico complejo inducido por fuerzas biomecánicas que suelen manifestarse como una disfunción neurológica transitoria que resuelve espontáneamente en el tiempo y puede no involucrar pérdida de consciencia (2). Sus indicadores son: (1)

o Desorientación o confusión documentada inmediatamente después del evento.

o Deterioro del equilibrio el día después del evento.

- Disminución de la velocidad de respuesta 2 días posterior al trauma.

o Alteración de la memoria y aprendizaje en los dos días posteriores al trauma.

\section{Clínica}

En general, un TCE puede desencadenar los siguientes síntomas (9): períodos de pérdida o disminución de la consciencia, pérdida de memoria de eventos inmediatamente antes o después del trauma, alteración del estado mental como confusión, desorientación y bradipsiquia, déficit neurológico como debilidad y pérdida 


\section{CIENCIA\&SALUD}

del equilibrio.

En una contusión cerebral se pueden encontrar las siguientes características: ausencia de pérdida de consciencia que dure menos de 6 horas, ausencia o leve déficit motor y amnesia post-traumática de minutos a horas o ausencia de alteración de la memoria (9).

Algunos pacientes presentan el Síndrome Post Concusivo el cual se define como la persistencia de síntomas como falta de concentración, mareos, cansancio, cefalea, irritabilidad, trastornos depresivos y trastornos del sueño; que suelen durar un promedio de 90 días (10). Sin embargo, la mayoría de las personas muestra resolución de sus síntomas dentro de los primeros 7 a 10 días (11).

El Síndrome Post Concusivo (3) es un concepto clínico difícil debido a que es su diagnóstico queda atado a la definición actual de TCE leve. Principalmente, no existe consenso en cuanto a que el síndrome sea inmediatamente posterior a un TCE leve o hasta 3 meses después. La ClE-10 (Clasificación Internacional de Enfermedades en su décima edición) propuso en 1992 que un síndrome post concusivo se define como:

- Un síndrome que sigue a un trauma craneoencefálico.

- Al menos 3 de los siguientes síntomas:

\begin{tabular}{|c|c|c|c|}
\hline Cefalea & Mareos & Fatiga & $\begin{array}{c}\text { Dificultad para la } \\
\text { concentración }\end{array}$ \\
\hline Irritabilidad & $\begin{array}{c}\text { Compromiso de la } \\
\text { memoria }\end{array}$ & Insomnio & $\begin{array}{c}\text { Intolerancia al } \\
\text { estrés, emociones } \\
\text { y alcohol }\end{array}$ \\
\hline
\end{tabular}

El DSM-IV (Manual diagnóstico y estadístico de los trastornos mentales) define al Síndrome post concusivo adicionalmente a lo anterior, el compromiso en la concentración o memoria que esté presente en pruebas neuropsicológicos, y que los síntomas sean fuente de impedimento significativo en funcionamiento social u ocupacional por al menos tres meses.

Los criterios de la ClE-10 se enfocan en síntomas que hacen más vulnerable el diagnóstico en personas con desórdenes como depresión o ansiedad.

La Organización Mundial de la Salud concluyó que tanto el CIE-10 como el DMS-IV no tenían sustento empírico debido a que los síntomas de ambos criterios son poco específicos para el TCE leve.

Los síntomas posteriores a una concusión caen en tres categorías: somáticos, cognitivos y emocionales, sin embargo todos los anteriores son poco específicos en cuanto a que pueden aplicar a muchos cuadros neuropsiquiátricos y son poco predecibles.

EI DMS-IV define al síndrome post concusivo como un déficit cognitivo más síntomas en 3 de 8 dominios, aunque 5 de ellos podrían ser causa directa del déficit cognitivo requerido para el diagnóstico de concusión: fatiga, insomnio, cefalea, ansiedad, depresión, apatía. La validez y especificidad del síndrome post concusivo se queda corto en cuanto a diagnosticar otras enfermedades concomitantes, como el dolor, estrés, ansiedad, desórdenes del sueño, depresión.

Los clínicos deben distinguir claramente entre la entidad similar en cuanto a sintomatología que es el trastorno de estrés post trauma.

\section{Criterios para imagen}

Inicialmente, al valorar un paciente con trauma craneoencefálico, se debe utilizar la Escala de Coma de Glasgow (GCS). La misma tiene cuatro décadas de uso sin modificaciones; y valora el grado de afectación del nivel de consciencia de un paciente tras un traumatismo craneoencefálico. 
La mayoría de las tomografías de sistema nervioso central (SNC) no tienen hallazgos radiológicos, sin embargo, la Regla de Tomografía de SNC de Canadá (Canadian Head CT Rule) se desarrolló para resolver este dilema (12). La misma es una escala que se debe aplicar únicamente a pacientes con historia de trauma craneoencefálico con un GCS de entre 13 y 15, que hayan presentado alguno de los siguientes: (12)

- Pérdida de consciencia

- Amnesia del evento de trauma

- Desorientación presenciada

Los criterios de exclusión son los siguientes:

- Edad menor a 16 años

- Uso de anticoagulantes o antiagregantes

- Convulsión posterior a trauma

La escala canadiense toma en cuenta criterios de mediano y alto riesgo. Los pacientes que cumplan criterios de alto riesgo pueden requerir intervención neuroquirúrgica. Los anteriores son: (12)

Regla de Tomografía de SNC de Canadá:

\begin{tabular}{|c|c|}
\hline Criterios de mediano riesgo & Criterios de alto riesgo \\
\hline Amnesia retrógrada al evento mayor a 30 minutos & Glasgow menor a 15 dos horas posteriores al trauma \\
\hline \multirow{4}{*}{$\begin{array}{l}\text { Mecanismo de alto riesgo: peatón colisionado por } \\
\text { vehículo, ocupante de vehículo que fue expulsado del } \\
\text { mismo, caída de entre } 1 \text { y } 1.5 \text { metros }\end{array}$} & $\begin{array}{c}\text { Sospecha de fractura abierta o deprimida de porción } \\
\text { basilar del cráneo }\end{array}$ \\
\hline & $\begin{array}{c}\text { Signos y síntomas de sospecha de fractura basilar (ojos } \\
\text { de mapache, hemotímpano, signo de Battle, otorrea o } \\
\text { rinorrea). }\end{array}$ \\
\hline & Dos o más episodios de vómitos \\
\hline & Edad mayor a 65 años \\
\hline
\end{tabular}

Si el paciente no cumple con los criterios mencionados, no es necesario realizar una tomografía. La escala tiene una sensibilidad del $83-100 \%$ para detectar lesiones intracraneales traumáticas y detecta 100\% de los pacientes que requieran intervención neuroquirúrgica (12).

La escala de Nueva Orleans para TC de SNC ha demostrado en un estudio que ha sido más sensible en detectar trauma intracraneal, sin embargo, es menos específica que la escala canadiense (12). Esta toma en cuenta a un paciente con GCS menor a 15 y mayor a 13:

- Cefalea

- Vómitos

- Edad > 60 años

- Intoxicación por alcohol/drogas

- Amnesia anterógrada persistente

- Trauma visible por encima de la clavícula.

En el estudio canadiense, de 3121 adultos con TCE leve, 8\% tenía una lesión cerebral clínicamente importante (con evidencia de hallazgo agudo en tomografía que requiera admisión o revaloración neurológica). Un $1 \%$ requería intervención neuroquirúrgica y $4 \%$ tenía lesiones de poca importancia (sobre todo pequeños hematomas subaracnoideos o contusiones que no requieren intervención, determinados como lesiones 


\section{CIENCIA\&SALUD}

insignificantes; y que adicionalmente son asintomáticos en valoraciones subsecuentes) (12).

Adicionalmente, los investigadores encontraron cinco hallazgos de alto riesgo: fallo en alcanzar un GCS de 15 a las 2 horas de presentación, sospecha de fractura abierta, signos de fractura de base de cráneo, vómitos mayores a dos episodios y edad mayor a 65 años. Un solo factor de riesgo alto da un 100\% de sensibilidad en pacientes que necesitan intervención neuroquirúrgica.

\section{Uso de neuroimagen avanzada (Resonancia Magnética):}

La Resonancia Magnética (RM) es 25-30\% más sensible que la TC en revelar lesiones por cizallamiento. Las modalidades funcionales como la tomografía por emisión de positrones (SPECT) tienen mayor probabilidad de evidenciar anomalías en pacientes por quejas persistentes (mayores a 1 año) somáticas y cognitivas. La resonancia magnética funcional ha permitido observar la relación entre el trauma craneoencefálico leve y la función cerebral. En un estudio en jugadores de fútbol americano, la resonancia magnética funcional evidenció aumento de la activación en la corteza motora y promotora, parietal superior e inferior, así como cerebelar en la semana posterior a la lesión.

En cuanto a pacientes pediátricos, el Pediatric Emergency Care Applied Research Network (PECARN) resolvió diferenciar entre los pacientes pediátricos de bajo riesgo por trauma craneoencefálico leve a aquellos con trauma clínicamente significativo. Este algoritmo indica no realizar TC en pacientes con estado mental normal, sin pérdida de consciencia, vómitos, mecanismo de trauma no severo, sin signos de fractura de base de cráneo y cefalea severa. Su valor predictivo negativo es de $99.5 \%$, con cero intervenciones neurológicas pasadas por alto (12). Como dato adicional, los movimientos extraoculares patológicos en paciente pediátrico están presentes en hasta $22 \%$ de los TCE leves (11).

En el paciente pediátrico que tiene una alta incidencia de TCE se debe alertar a los cuidadores sobre síntomas para reconsultar, entre ellos: (13)

- Cefalea severa, que empeora

- Convulsiones

- Dolor cervical severo

- Obnubilación, dificultad para mantener alerta

- Vómitos repetidos

- Disartria

- Dificultad para reconocer lugares o personas

- Confusión que empeora

- Debilidad o anestesia en miembros superiores o inferiores

- Cambios en el comportamiento inusuales

- Irritabilidad

- Pérdida de consciencia

\section{Criterios para ingreso}

No existe un consenso para saber cuáles pacientes necesitan una TC de seguimiento entre aquellos que presentan lesión intracraneal tras un TCE leve. Usualmente, se mantiene el paciente en observación por al menos 24 horas con el fin de descartar progresión del sangrado intracraneal y obtener evidencia radiológica 
indicativa de necesidad de intervención quirúrgica, ingreso hospitalario o alta (14). En un meta-análisis reciente se observó que la tomografía programada conlleva a cambios en el manejo en un grupo pequeño de pacientes $(9,6-11,4 \%)(14)$.

El criterio más importante a tomar en cuenta para el ingreso hospitalario para la TC control es el tipo de lesión intracraneal. La hemorragia subaracnoidea de la convexidad, hematomas subdurales laminares o hematomas de escaso volumen $(<4-7 \mathrm{~mm})$, así como pequeñas contusiones únicas de la convexidad. Entre los anteriores se debe tomar en cuenta adicionalmente la evolución y situación clínica del paciente, como la presencia de coagulopatía u otras discrasias sanguíneas que puedan progresar la lesión (14).

El mecanismo lesional de alta energía, el tratamiento antiagregante asociado a un nivel excesivo de anticoagulantes (con un INR > 3) son puntos para tomar en cuenta durante la decisión de internar un paciente. Igualmente, a la hora de ingresar, son importantes los pacientes con derivaciones ventrículo peritoneales para controlar la hipertensión intracraneal (14).

En conclusión, según los lineamientos actuales, entre los pacientes con lesiones intracraneales que no son de manejo quirúrgico, el riesgo de sangrado tardío es lo suficientemente bajo como para permitir el alta hospitalaria con las recomendaciones del caso (14). Es decir, pacientes con una puntuación en la Escala de Glasgow de 15 puntos sin otros factores de riesgo, deberían ser dados de alta sin TC ni observación y con recomendaciones específicas (5).

Vale la pena recalcar que una segunda concusión que ocurre minutos o días posterior a la primera, conocida como Síndrome de Segundo Impacto, se asocia a síntomas más floridos y recuperación prolongada; así como muerte súbita y deterioro catastrófico neurológico en una minoría de los pacientes. Seguidamente a un trauma craneoencefálico, según un modelo animal, existe una ventana de vulnerabilidad metabólica. Un solo TCE leve resulta en un descenso significativo en el metabolismo cerebral de glucosa, que retorna a la normalidad a los tres días. Si se produce un nuevo traumatismo 24 horas después del primero, se suprime aún más este metabolismo, lo cual resulta en una recuperación más prolongada (12).

\section{Manejo inicial}

Para individuos tanto de manejo ambulatorio como de ingreso, se debe restringir la actividad física y cognitiva por las primeras 24 a 72 horas del TCE leve, dado a que los síntomas pueden empeorar cuando se desafía el desajuste metabólico (7). Posterior a ello, se pueden retomar actividades que no van a exacerbar síntomas. Se debe tener un monitoreo cercano en caso de que hallan alteraciones neurológicas, principalmente en niños. Para los adultos, los síntomas de déficit cognitivo y balance van a reestablecerse para las dos semanas posteriores. Se ha observado que, en adolescentes y adultos jóvenes, los antecedentes de patología psiquiátrica y de cefalea tipo migrañosa tienden a persistir con síntomas por un período mayor al mes (15).

Los pacientes con TCE que presentan contusión, hematomas extra axiales y daño axonal difuso, deben ser monitorizados cuidadosamente. Su manejo inicial debe incluir: (1)

1. Posición Semi-fowler, o elevación de la cabecera a 30 grados ya que disminuye la presión intracraneal por intercambio del líquido cefalorraquídeo desde compartimientos intracraneales, así como promoción del flujo venoso.

2. Hiperventilación: mediante la reducción de la presión parcial de dióxido de carbono, se activa la vasoconstricción, lo cual reduce el volumen sanguíneo cerebral. Se debe utilizar solo en períodos breves.

3. Prevención de convulsiones: las guías actuales indican la utilización de una semana de anticonvulsivantes profilácticos.

4. Terapia hiperosmolar: se puede utilizar en forma de infusión o en bolo. El manitol, además de ser un diurético osmótico, tiene efecto en la viscosidad de la sangre, aumentando el volumen del LCR 


\section{CIENCIA\&SALUD}

y en respuesta autorregulatoria se da vasoconstricción, que últimamente limita el flujo del líquido y disminuye la presión intracraneal (PIC).

5. Coma medicamentoso: se utiliza midazolam o pentobarbital en infusión. Su mecanismo es la disminución significativa de la demanda metabólica del cerebro. Usualmente se utiliza posterior a que se han tomado medidas invasivas para monitorizar la presión intracraneal.

6. Enfriamiento terapéutico: se ha evidenciado que el estrés oxidativo es un efecto secundario al TCE. En niños se ha demostrado que disminuye la injuria oxidativa. Se deben monitorizar las alteraciones que tiene la temperatura en la glicemia, plaquetas y factores de coagulación. Ha tenido resultados mixtos y se considera una terapia secundaria.

7. Monitoreo de PIC: se utiliza un drenaje ventricular externo, que dependiendo de la lesión presentada tiene tanto propósito terapéutico como diagnóstico.

\section{CONCLUSIÓN}

El trauma craneoencefálico leve es una entidad de suma importancia en los servicios de emergencias del mundo, ya que es un motivo de consulta muy común. Su diagnóstico certero es clave en el manejo adecuado de aquellos pacientes que requieran intervenciones quirúrgicas. Las poblaciones especiales como los niños y los adultos mayores, se deben evaluar cuidadosamente debido a que tienen un comportamiento fisiológico distinto. El adecuado manejo y diagnóstico del trauma craneoencefálico leve es fundamental para la prevención de consecuencias letales e incapacitantes en la población general.

No se dieron conflictos de intereses ni hubo financiamiento para el presente artículo.

\section{REFERENCIAS BIBLIOGRÁFICAS}

1. Ricker JH, Arenth PM. Traumatic brain injury. Funct MRI Appl Clin Neurol Psychiatry. 2006;26(7):197-206.

2. Muñoz-Santanach D, Trenchs Sainz de la Maza V, Maya Gallego S, Cuaresma González A, Luaces Cubells C. Clinical observation: A safe alternative to radiology in infants with mild traumatic brain injury. An Pediatr. 2017;87(3):164-9.

3. Sussman ES, Pendharkar A V., Ho AL, Ghajar J. Mild traumatic brain injury and concussion: terminology and classification [Internet]. 1st ed. Vol. 158, Handbook of Clinical Neurology. Elsevier B.V.; 2018. 21-24 p. Available from: http://dx.doi.org/10.1016/B978-0-444-63954-7.00003-3

4. Valle Alonso J, Fonseca del Pozo FJ, Vaquero Álvarez M, Lopera Lopera E, Garcia Segura M, García Arévalo R. Comparación de la regla canadiense y los criterios de New Orleans en el traumatismo craneal leve en un hospital español. Med Clin (Barc) [Internet]. 2016;147(12):523-30. Available from: http://dx.doi.org/10.1016/j.medcli.2016.07.024

5. Freire-Aragón MD, Rodríguez-Rodríguez A, Egea-Guerrero JJ. Update in mild traumatic brain injury. Med Clin (Barc) [Internet]. 2017;149(3):122-7. Available from: http://dx.doi.org/10.1016/j. medcli.2017.05.002

6. Zufiría JMO, Prieto NL, Cuba BC, Degenhardt MT, Núñez PP, Serrano MRL, et al. Traumatismo craneoencefálico leve Mild head injury. Surg Neurol Int. 2018;9:S16-28.

7. Lumba-Brown A, Yeates KO, Sarmiento K, Breiding MJ, Haegerich TM, Gioia GA, et al. Centers for Disease Control and Prevention Guideline on the Diagnosis and Management of Mild Traumatic 


\section{CIENCIA\&SALUD}

Brain Injury among Children. JAMA Pediatr. 2018;172(11):1-13.

8. Corwin DJ, Grady MF, Joffe MD, Zonfrillo MR. Pediatric Mild Traumatic Brain Injury in the Acute Setting. Pediatr Emerg Care. 2017;33(9):643-9.

9. Pervez M, Kitagawa RS, Chang TR. Definition of Traumatic Brain Injury, Neurosurgery, Trauma Orthopedics, Neuroimaging, Psychology, and Psychiatry in Mild Traumatic Brain Injury. Neuroimaging Clin N Am [Internet]. 2018;28(1):1-13. Available from: https://doi.org/10.1016/j.nic.2017.09.010

10. Vacca VM. Tratamiento del traumatismo craneoencefálico leve en adultos. Nurs (Ed española) [Internet]. 2019;36(2):32-9. Available from: https://doi.org/10.1016/j.nursi.2019.03.010

11. Whitney SL, Sparto PJ. Eye Movements, Dizziness, and Mild Traumatic Brain Injury (mTBI): A Topical Review of Emerging Evidence and Screening Measures. J Neurol Phys Ther. 2019;43(April):S31-6.

12. Kamins J, Giza CC. Concussion-Mild Traumatic Brain Injury: Recoverable Injury with Potential for Serious Sequelae. Neurosurg Clin N Am [Internet]. 2016;27(4):441-52. Available from: http:// dx.doi.org/10.1016/j.nec.2016.05.005

13. Almeida AA, Lorincz MT, Hashikawa AN. Recent Advances in Pediatric Concussion and Mild Traumatic Brain Injury. Pediatr Clin North Am [Internet]. 2018;65(6):1151-66. Available from: https:// doi.org/10.1016/j.pcl.2018.07.006

14. Freire-Aragón MD, Rodríguez-Rodríguez A, Egea-Guerrero JJ. Update in mild traumatic brain injury. Med Clin (Barc). 2017;149(3):122-7.

15. Marklund N, Bellander BM, Godbolt AK, Levin H, McCrory P, Thelin EP. Treatments and rehabilitation in the acute and chronic state of traumatic brain injury. J Intern Med. 2019;285(6):60823. 